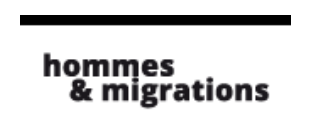

Hommes \& migrations

Revue française de référence sur les dynamiques

migratoires

1286-1287 | 2010

Les migrations subsahariennes

\title{
Partir pour aider ceux qui restent ou la dépendance face aux migrations
}

L'exemple des paysans sahéliens

\section{Florence Boyer et Harouna Mounkaila}

\section{(2) OpenEdition \\ 1 Journals}

\section{Édition électronique}

URL : http://journals.openedition.org/hommesmigrations/1752

DOI : 10.4000/hommesmigrations. 1752

ISSN : 2262-3353

Éditeur

Musée national de l'histoire de l'immigration

\section{Édition imprimée}

Date de publication : 1 juillet 2010

Pagination : 212-220

ISSN : 1142-852X

\section{Référence électronique}

Florence Boyer et Harouna Mounkaila, « Partir pour aider ceux qui restent ou la dépendance face aux migrations », Hommes \& migrations [En ligne], 1286-1287 | 2010, mis en ligne le 29 mai 2013, consulté le 19 avril 2019. URL : http://journals.openedition.org/hommesmigrations/1752 ; DOI : 10.4000/

hommesmigrations. 1752 


\section{Partir pour aider ceux qui restent ou la dépendance face aux migrations L’exemple des paysans sahéliens}

Par Florence Boyer, géographe, UMR 201 " Développement et Sociétés", université de Paris I Panthéon-Sorbonne, Institut de Recherche pour le Développement et Harouna Mounkaila, géographe, École normale supérieure, université Abdou Moumouni, Niamey

Dans les régions sahéliennes, le sort des paysans est étroitement lié

à leur mobilité spatiale. Les pénuries alimentaires fréquentes et la croissance démographique poussent de nombreux hommes à migrer

de manière temporaire afin de pallier les déficiences de l'agriculture villageoise. Leur absence est rythmée par le type de culture agricole mis

en place dans les villages, car c'est de lui que dépend la capacité de financement de la migration. Ces mobilités circulaires dévoilent ainsi une relation d'interdépendance inédite avec leurs espaces de départ. 
Les migrations saisonnières de travail sont, depuis quasiment un siècle, une pratique spatiale fondamentale pour la compréhension des espaces ruraux sahéliens, de leurs modes d'organisation sociale et économique. En effet, l'alternance entre une saison des pluies d'une durée de trois mois et une saison sèche favorise le développement d'activités complémentaires au cours de cette même saison. Pendant une période de huit à neuf mois, la main-d'ceuvre masculine est ainsi libérée des obligations de l'agriculture pluviale. L'émergence de ces migrations temporaires de travail est à mettre en relation avec l'installation de l'administration coloniale qui a conduit non seulement à une pacification de certaines régions, mais qui a surtout imposé le paiement de l'impôt et les travaux forcés. La monétarisation de l'économie a largement favorisé le développement de ces mouvements, qui se sont dirigés dans un premier temps vers les pays côtiers, plus particulièrement le Ghana, le Nigeria et la Côte d'Ivoire ${ }^{(1)}$.Si, au début, l'obligation du paiement de l'impôt et les travaux forcés ont motivé les départs, progressivement, le besoin d'acquérir certains biens marchands, au premier rang desquels les vêtements, les chaussures, le savon, a favorisé l'expansion de ces mouvements saisonniers qui concernent quasi uniquement les hommes. Ils ont donné lieu dans certains cas à des installations définitives ${ }^{(2)}$ dans les grandes villes côtières du Golfe de Guinée. Dans la période plus récente, à partir des années quatre-vingt, les pratiques migratoires vont connaître des changements importants en relation avec les dynamiques de l'espace local. Dans ces lieux de départ, la croissance démographique forte accroît la pression foncière et participe à la libération d'une main-d'ceuvre qui a davantage la possibilité de se consacrer à des activités extra-agricoles ; parallèlement et depuis une trentaine d'années, la baisse et l'irrégularité des précipitations ont conduit à une diminution des récoltes et à l'installation d'une disette structurelle dans nombre de régions.

Dans un tel contexte, les temporalités migratoires se sont adaptées, c'est-à-dire qu'elles ont perdu leur caractère régulier: les migrants sont absents pendant des durées variant de une à plusieurs années et surtout leur retour ne correspond plus systématiquement à la saison des pluies. Toutefois, ce schéma classique des migrations circulaires perdure et les mouvements restent encore l'apanage des hommes.

\section{Une mobilité génératrice d'équilibre}

Le caractère massif des migrations circulaires, associé aux difficultés grandissantes de séjourner dans certains pays, a conduit également à une diversification des destinations. À l'heure actuelle, les espaces urbains concernés ne se limitent plus au Golfe de Guinée. Les migrants circulaires partent jusqu'à Dakar ou en Afrique 
centrale et depuis les années soixante-dix jusqu'en Libye et en Algérie (en lien avec l'exploitation pétrolière dans ces deux pays); il ne faut pas oublier non plus le maintien des migrations internes à des niveaux relativement élevés.

Notre objectif est de questionner ce schéma d'organisation contemporain en interrogeant, d'une part, les dynamiques des systèmes de production locaux, et, d'autre part, celles des migrations circulaires. Pour cela, nous postulons qu'il est plus pertinent d'observer les dynamiques sociales et économiques, non pas comme l'expression d'un système de survie, point de vue souvent adopté pour décrire les sociétés rurales sahéliennes, mais plutôt comme la traduction d'un équilibre qui permet aux populations de rester installées dans les villages, c'est-à-dire qui leur permet aussi d'être immobiles.

La région de Tahoua, située dans le massif de l'Ader, au centre-ouest du Niger, au niveau de la limite des cultures sous pluies, est souvent présentée comme l'une des principales régions de départ de ce pays pour ce qui est des migrations circulaires. Dans cette région majoritairement haoussa, qui abrite aussi des populations touarègues et peuhles, la culture du mil constitue l'activité agricole fondamentale, en association avec l'élevage de petits ruminants et de bovins, mais de façon moindre; s'y ajoutent les cultures de contre-saison, notamment de tomates et d'oignons, qui se développent surtout dans les vallées. En 2008, a été menée une enquêtete dans cette région visant à mesurer l'ensemble des formes de mobilité, c'est-à-dire les migrations internes et internationales à différentes échelles temporelles (saisonnière, annuelle, biographique). L'objectif est de se donner les moyens de mesurer et de décrire les circulations qui participent des dynamiques de ce territoire, en tenant compte de leurs temporalités diverses. Ces migrations ont été replacées dans leur contexte villageois, familial et individuel. Cette enquête a été conduite dans quatre départements de la région (ceux d'Illéla, Tahoua, Keita et Bouza), laissant ainsi de côté la grande zone pastorale du nord et de l'ouest de la région moins concernée par les migrations circulaires.

\section{Des migrations massives aux temporalités propres}

Quelle que soit la situation de ces villages, c'est-à-dire qu'ils aient la possibilité ou non de pratiquer des cultures maraîchères destinées à la vente, tous sont massivement concernés par les migrations circulaires de travail qui sont, exclusivement ou presque, le fait d'hommes jeunes entre 15 et 40 ans environ. L'analyse des biographies migratoires a permis de montrer qu'à l'âge de 30 ans, la 
moitié des hommes ont effectué au moins une migration ; cependant, dès l'âge de 15 ans, le quart d'entre eux a déjà migré au moins une fois hors de son village. Audelà du caractère massif et précoce des migrations, il apparaît que ce premier départ enclenche les autres qui se succèdent tout au long de la vie active de l'individu, jusque vers l'âge de 40 ans. À l'échelle de la vie des individus, à l'âge où les hommes partent en migration, les femmes se marient, s'installent chez leurs beaux-parents et fondent leur foyer avec la présence alternante de leur mari.

Les migrations circulaires restent ainsi un phénomène masculin et elles sont marquées par la jeunesse de ceux qui migrent ; si les carrières migratoires ne sont pas normées en termes de parcours, nombre d'entre eux débutent par une ou plusieurs migrations internes qui les mènent soit vers les villes minières de l'uranium et du charbon (Arlit ou Tchirozérine), soit vers la capitale, Niamey. Les mouvements vers le nord du Nigeria (Kano, Jos...) peuvent être assimilés à ces migrations internes, étant donné autant la proximité de ces villes, que la longue histoire commerciale et migratoire qui lie ce pays à la région de Tahoua. Une fois ces premières expériences de la migration et de l'urbain acquises, l'éventail des destinations possibles s'élargit et surtout devient plus lointain: sont concernées ainsi les grandes villes du Ghana, de Côte d'Ivoire, du Sénégal,

D'une certaine manière,
la dispersion, continue
à l'échelle villageoise,
passagère au niveau
individuel, joue un rôle
dans la possibilité
de maintenir la présence
et la cohésion sociale
au niveau local.
du Cameroun ou du Gabon, mais aussi certaines villes de Libye et d'Algérie. La diversification des destinations au fil des décennies répond, d'une part, aux difficultés grandissantes d'insertion professionnelle dans nombre de villes de la sous-région, et, d'autre part, aux difficultés de circulation et d'installation même temporaire dans certains pays. Par ailleurs, un autre avantage de cette diversité de lieux possibles est qu'en cas de crise, d'impossibilité de circuler dans l'un ou l'autre des pays, les migrants ont la capacité de se replier ailleurs.

L'importance des migrations circulaires, associées à des filières migratoires établies et diversifiées, font de ces mouvements une pratique fondamentale dans l'organisation des villages de la région de Tahoua. En effet, une grande partie de la main-d'ceuvre masculine est absente pendant plusieurs mois, voire plusieurs années ; cette maind'ceuvre ne peut donc pas participer chaque année aux travaux des champs.

Une observation plus fine des temporalités des migrations circulaires, à l'échelle de l'année, permet de mettre en évidence le lien entre cette pratique de l'absence, les rythmes de la migration et les systèmes d'organisation et de production locaux et surtout la manière dont ces absences sont gérées. Au niveau des trois départements 
enquêtés, 5 \% de la population active masculine est absente tout au long de l'année, c'est-à-dire qu'elle ne participe pas aux activités agricoles et d'élevage ; on atteint un pic de $20 \%$ de la population masculine absente au cceur de la saison sèche, c'est-à-dire entre les mois de décembre et d'avril. Si l'alternance classique entre saison sèche et saison des pluies se retrouve de façon assez notable, il apparaît que l'agriculture n'emploie pas la totalité de la main-d'ceuvre masculine. Le contexte de croissance démographique et la pression foncière qui s'ensuit - qui a été relevé par les habitants de nombre de villages - conduisent à libérer une partie de la main-d'ceuvre disponible. Cependant, si celle-ci ne s'emploie plus régulièrement à l'échelle locale, elle reste impliquée à ce niveau au sens où les retours persistent, mais selon des temporalités déconnectées du village. Certes, la présence des épouses, des enfants, concourt à ces retours périodiques, mais transparaît surtout une dynamique particulière dans ce lien entre migrations circulaires et organisation sociale et économique villageoise.

\section{Une pratique de l'absence qui sert l'unité du groupe}

Si les temporalités des migrations circulaires sont en partie déconnectées des rythmes saisonniers de l'économie agricole villageoise, il n'en reste pas moins que la persistance des allers-retours dénote un ancrage autant des migrants que des migrations dans ces villages. Leur présence est requise périodiquement pour assurer la reproduction familiale et la pérennité de cette famille au niveau local, mais aussi sur un plan économique, pour que l'ensemble du groupe bénéficie des migrations et de ses revenus. D'une certaine manière, la dispersion, continue à l'échelle villageoise, passagère au niveau individuel, joue un rôle dans la possibilité de maintenir la présence et la cohésion sociale au niveau local.

Une observation fondée sur les systèmes de production villageois permet de préciser les liens entre la dispersion temporaire et l'installation à l'échelle locale. En effet, il apparaît qu'une part des revenus de l'économie agricole est investie dans la migration, c'est-à-dire pour le voyage et la création d'une activité par les migrants. Toujours à l'échelle des mobilités de l'année, une distinction importante est présente entre les villages dont le système de production est fondé uniquement sur le mil - avec éventuellement un élevage de petits ruminants - et les villages dont le système de production est fondé sur l'association entre culture du mil et culture de contre-saison (avec ou sans élevage ${ }^{(4)}$. Alors que la production de mil est quasi totalement destinée à la consommation familiale, quel que soit le profil des villages, 
la production maraîchère d'oignons et de tomates - qui ne concerne que $12,4 \%$ des exploitations agricoles de la zone - est destinée à la vente, permettant de dégager un revenu annuel.

Sur le plan des migrations circulaires, les individus appartenant à l'un ou l'autre de ces profils d'exploitation n'ont pas les mêmes pratiques. Pour ceux qui relèvent d'exploitations où seule la culture du mil est pratiquée, les départs et les retours sont très largement rythmés par l'alternance des saisons, et ainsi par l'obligation de cultiver. La part de la population absente toute l'année est moindre. Également, les destinations privilégiées sont proches, essentiellement Niamey et le Nigeria.

Les villages qui pratiquent des cultures maraîchères en complément du mil présentent un profil migratoire différent ; quasiment $10 \%$ de la population masculine est absente tout au long de l'année et surtout l'alternance entre saison sèche et saison des pluies ne transparaît que de façon assez marginale au niveau du rythme des migrations. Les destinations privilégiées sont non seulement plus diversifiées, mais aussi plus lointaines : elles concernent l'ensemble de l'Afrique de l'Ouest, une partie de l'Afrique centrale ainsi que l'Algérie et la Libye.

Cette distinction permet de mettre en évidence des modes d'organisation des circulations, et des modes de gestion du risque et de l'absence au niveau local ${ }^{(5)}$. En effet, lorsque la culture du mil est la seule ressource familiale, le risque de disette est accru et, surtout, il est quasiment impossible de dégager un revenu qui serait soit dépensé localement, soit investi dans la migration. La proximité des lieux de destination témoigne de l'impossibilité des migrants de financer un voyage plus lointain, qui serait aussi plus lucratif au retour. Au contraire, le revenu dégagé de la production maraîchère ne semble pas investi localement mais plutôt dans le financement d'une migration de relativement longue durée, vers des villes où l'insertion professionnelle est plus facile et surtout plus bénéfique. Apparaît aussi une spécialisation accrue de la main-d'ceuvre au sens où certains se destinent à l'agriculture alors que d'autres migrent, selon des rythmes déconnectés des saisons. L'observation du lien entre les systèmes de production locaux et l'organisation des migrations circulaires montre comment le risque est géré localement, et surtout comment cette zone a réussi à s'extraire d'une logique de survie malgré les pénuries alimentaires récurrentes. Quelle que soit la situation, les migrations circulaires sont totalement parties prenantes du système d'organisation sociale et économique. Si l'absence est encouragée et nécessaire, elle n'empêche pas non plus la pratique de l'agriculture et surtout, ne conduit pas à des départs définitifs. Au contraire, la migration apporte les ressources que ne peut plus fournir l'agriculture, permettant justement de pérenniser les systèmes locaux. Le risque est géré par le maintien de cet équilibre économique et social entre agriculture et migration. 


\section{De la dépendance du local face aux migrations}

L'une des conditions pour que ce système fonctionne et qu'un certain équilibre se maintienne est que les migrants envoient, ou ramènent avec eux, de l'argent et des biens à leur famille. Une évaluation des remises à partir des banques, de Western Union et des comptoirs en relation avec la Libye installés dans la ville de Tahoua a montré que plusieurs milliards de francs CFA parviennent chaque année dans cette région. Certes, ces remises ne sont pas le seul fait des migrants circulaires ${ }^{(6)}$, de même que ne sont pas pris en compte ce que les migrants ramènent avec eux lors de leur retour ou ce qu'ils confient à des ressortissants du même village.

L'analyse de l'usage de ces remises au niveau villageois a montré qu'elles sont investies essentiellement dans l'achat de marchandises courantes, au premier rang desquels les achats de nourriture, de vêtements, de chaussures, de savons... Les investissements dans la construction, dans l'amélioration des techniques culturales, dans la création d'activités (commerce, artisanat...) sont plus limités. Lobjectif principal des revenus issus des migrations circulaires est ainsi de pallier les déficiences de l'agriculture, c'est-à-dire de permettre aux populations d'assurer leurs besoins vitaux tout au long de l'année. Apparait, en fait un mécanisme de dépendance entre les populations migrantes et ceux qui ne bougent pas - les femmes, les enfants - ou ceux qui ont cessé de bouger. D'une certaine manière, sans les premiers, les seconds se verraient contraints à la mobilité au sens où l'espace local n'est pas à même de leur fournir les moyens de rester sur place tout au long de l'année. Autrement dit, la mobilité de certains permet non seulement l'immobilité des autres, mais aussi la durabilité de l'installation de chacun dans le village.

Cependant, il convient d'observer de plus près ce mécanisme de dépendance apparu avec la migration. Étant donné l'usage qui est fait des revenus de la migration, il apparait pertinent de comparer les effets produits par cet apport et ceux que peuvent produire les apports de l'aide. Il a été montré l'existence d'une dépendance dynamique entre l'aide et les bénéficiaires, au sens où "l'aide appelle l'aide ${ }^{(7)}$. Ce mécanisme se retrouve ici notamment dans le caractère irréversible des apports de la migration pour les populations immobiles, dans la mesure où ceux-ci ont introduit un niveau de ressources qui dépasse la capacité des villages à le reproduire. Ainsi, les revenus de la migration ne peuvent s'arrêter, voire même ne peuvent diminuer : ce type de relation pose la question des conditions de l'accueil des migrants dans leurs lieux de destination. En effet, ils restent soumis aux aléas des politiques migratoires, aux expulsions régulières de certains pays (Gabon, Libye...), aux crises qui émaillent périodiquement la stabilité d'autres (Côte d'Ivoire, Nigeria...). Si la diversité des 
destinations possibles contribue à diminuer le risque présenté par un lieu d'accueil unique, il n'en reste pas moins que les migrants peuvent se retrouver momentanément sans la capacité d'envoyer quelque chose à leur famille.

Un autre phénomène que l'on prête généralement à l'aide et au mécanisme de dépendance qu'elle suscite est la "désincitation à l'effort due à la disponibilité de l'aide ${ }^{(8) "}$, c'est-à-dire qu'elle briserait les systèmes de production locaux et leurs dynamiques. La migration ne crée pas une telle logique de dépendance, au sens où ce sont justement les ressources locales qui servent à son financement; sans la vente d'une partie de la production agricole, les migrants ne pourraient pas partir et créer une activité temporaire en ville. Toutefois, sans aller jusqu'à la déstructuration des systèmes de production locaux, il arrive que ceux-ci ne soient pas à même de fournir les ressources indispensables au départ; les migrants ont alors la possibilité soit d'emprunter au départ le coût de leur voyage, soit de pratiquer une "arrivée-payé", c'est-à-dire que le transporteur

\section{Le mécanisme de dépendance qui apparaît entre les migrations et l'échelle locale est, au final, à double sens. Les départs ne sont autorisés que parce que le système de production les permet autant socialement qu'économiquement.} leur donne la possibilité de partir et les frais de transport seront remboursés à l'arrivée par les ressortissants du même village. Ces derniers fourniront, également sous forme de prêt, la somme nécessaire au lancement d'une activité. Ainsi, un bon fonctionnement du système de production villageois est requis pour que la migration ait lieu : l'investissement au niveau local permet de susciter une aide à moyen terme.

Le mécanisme de dépendance qui apparaît entre les migrations et l'échelle locale est, au final, à double sens. Les départs ne sont autorisés que parce que le système de production les permet autant socialement qu'économiquement. Et ce dernier subsiste et se maintient par l'aide régulière apportée par les circulations.

\section{Conclusion}

Le sort des paysans sahéliens apparaît plus que jamais lié à leur mobilité, c'est-à-dire à leur capacité à être autant des paysans que des migrants, à leur capacité à se maintenir à la fois dans l'agriculture et l'activité migratoire, à se disperser en permanence, mais pour revenir aussi vers leur village de départ en permanence. La 
subsistance des villages, dans un contexte de croissance démographique et de pénuries alimentaires fréquentes, repose sur le maintien de ces circulations et sur l'aide qu'elles apportent régulièrement. Si l'équilibre de ce système semble relativement précaire, dans la mesure où il est soumis autant à la poursuite de l'agriculture, qu'à la réussite en migration, il permet aux populations immobiles de rester sur place, d'éviter la contrainte d'un déplacement forcé.

Dans cette perspective, les migrations ne s'inscrivent pas dans une logique d'abandon des espaces de départ, mais contribuent plutôt au maintien de leur peuplement. À travers les pratiques migratoires, les paysans assument les contraintes des systèmes de production villageois et leur croissance démographique.

La migration est rarement une opportunité d'accumulation ou d'investissement dans l'agricole. À cet effet, l'on peut s'interroger sur son efficacité sur le long terme comme moyen de diversification des revenus contre la dépendance exclusive des activités rurales. Comment faire participer davantage les migrations dans la promotion de systèmes productifs plus intensifs et durables?

\section{Notes}

1. Voir Jean Rouch, Migrations au Ghana (Gold Coast). Enquête 1953-1955, Paris, Société des africanistes, 1956, p. 173 ; Jean Rouch, "Problèmes relatifs à l'étude des migrations traditionnelles et des migrations modernes en Afrique occidentale", Bulletin de l'IFAN, série B, t. 22, n 3-4, 1960, pp. 369-378 ; Jean-Pierre Olivier de Sardan, Les Sociétés songhay - zarma (Niger - Mali), Paris, Karthala, 1984, p. 299.

2. Les installations définitives dans certaines grandes villes des pays de la côte du Golfe de Guinée précèdent l'émergence massive des migrations saisonnières et sont plutôt à mettre en regard des relations commerçantes entre le Sahel et les pays du Golfe de Guinée.

3. Ce travail a été effectué dans le cadre du projet "Le Niger, espace d'émigration et de transit vers le nord et le sud du Sahara : rôle et comportement des acteurs, recompositions spatiales et transformations socio-économiques", in Document de synthèse des projets du programme FSP 2003-74 : "migrations internationales, recompositions territoriales et développement", Paris, IRD, 2009, pp. 109-120.

4. Nous laissons ici volontairement de côté les villages dont le système de production est fondé sur l'élevage transhumant de petits ruminants et de bovins. Historiquement les migrations circulaires y sont moins importantes, dans la mesure où cette activité requiert la présence permanente d'une grande partie de la main-d'ceuvre masculine. Lobservation du rythme annuel de ces villages montre des mouvements qui se font à l'intérieur de la région de Tahoua, selon un rythme lié aux animaux ; nous sommes face à des transhumants et non à des migrants.

5. André Quesnel, "De la communauté territoriale à l'organisation familiale en archipel : la mobilité spatiale des familles rurales en Afrique de l'Ouest et au Mexique", in Dureau Françoise et Hily Marie-Antoinette (éd.), Les mondes de la mobilité, Rennes, Presses Universitaires de Rennes, 2009, pp. 67-103.

6. Les migrants définitifs envoient aussi régulièrement de l'argent dans leurs villages d'origine ; il n'a pas été possible de distinguer les deux sources migratoires de ces remises.

7. Jean-David Naudet, "Le dilemme entre solidarité et dépendance", in Autrepart, n 13, 2000, pp. 173-193.

8. Idem. 
